\title{
Lexis
}

Journal in English Lexicology

Book reviews | 2018

\section{Laurie BAUER, Compounds and Compounding}

Cambridge University Press, 2017, 195 pages

Jean Missud

\section{OpenEdition}

Journals

Electronic version

URL: http://journals.openedition.org/lexis/2490

DOI: $10.4000 /$ lexis. 2490

ISSN: 1951-6215

\section{Publisher}

Université Jean Moulin - Lyon 3

\section{Electronic reference}

Jean Missud, «Laurie BAUER, Compounds and Compounding », Lexis [Online], Book reviews, Online since 06 December 2018, connection on 25 September 2020. URL : http://journals.openedition.org/lexis/ 2490 ; DOI : https://doi.org/10.4000/lexis.2490

This text was automatically generated on 25 September 2020.

\section{(c) (i) (9)}

Lexis is licensed under a Creative Commons Attribution-NonCommercial-NoDerivatives 4.0 International License. 


\title{
Laurie BAUER, Compounds and Compounding
}

Cambridge University Press, 2017, 195 pages

\author{
Jean Missud
}

\section{REFERENCES}

\section{Laurie Bauer}

Compounds and Compounding. Cambridge University Press, Cambridge Studies in Linguistics, Cambridge, 2017. ISBN : 978-1-108-40255-2, Prix : £19,99, 195 pages

Dans Compounds and Compounding, Laurie Bauer propose de répondre à certaines questions qui subsistent lorsque l'on s'intéresse au processus compositionnel anglais, et ce malgré le nombre important de travaux dédiés à ce sujet. Bauer s'intéresse à la question des mots composés depuis les années 1980. D’ailleurs, dans son introduction, Bauer note (p. xiii) que depuis sa thèse de doctorat soutenue en 1975, il n'a jamais cessé de travailler sur les mots composés. Il souligne surtout qu'au fil des décennies, son idée de ce qu'est un mot composé $\mathrm{a}$, de bien des manières, évolué, un élément qui le pousse à publier ce nouvel ouvrage sur la question. Dans Compounds and Compounding, il est notamment question des frontières entre mots et syntagmes et de l'étiquette qu'il serait préférable d'apposer sur le groupe des composés anglais, des relations entre les éléments qui forment un mot composé anglais, de la manière dont les différentes langues qui ont recours à la composition utilisent ce processus, et des travaux qui restent encore à mener en lien avec le processus compositionnel. L'ouvrage Compounds and Compounding aborde successivement toutes ces questions dans cinq chapitres: "Compounds and Words» (p.3), "The Grammar of Compounds» (p.29), "The Semantics of Compounds» (p.55), «The Classification of Compounds » (p. 107) et «Facets of English Compounding » (p. 126).

2 La première question abordée par Bauer est celle qui consiste à se demander si les mots composés anglais sont bel et bien à considérer comme des mots. L'argument utilisé par 
Bauer (3) est qu'il existe un lien entre les difficultés à formuler une définition de " mot » et les difficultés à formuler une définition de «mot composé ». Bauer avance l'idée selon laquelle le critère orthographique est une base instable pour définir à la fois «mot » et «mot composé ", car la manière dont un mot est orthographié est aléatoire (des questions de style, de choix éditoriaux ou de préférences personnelles entrent en jeu) : on trouvera par exemple aussi bien coffee-pot, coffee pot et coffeepot. À cela s'ajoute le problème que pose la consultation d'un dictionnaire : le fait qu'une entité soit ou ne soit pas listée dans un dictionnaire pourrait a priori être utilisé comme un critère. Or, Bauer (p.10) cite l'étude menée par Di Sciullo et Williams (1987) qui affirme le contraire. Le recours à un dictionnaire n'est donc pas pertinent pour déterminer si ce à quoi l'on a affaire est bien un "mot», a fortiori un «mot composé » dans le cas de structures utilisant plusieurs éléments. Bauer conclut ce premier chapitre sur le statut du terme « mot » en notant à la fois l'absence de consensus et l'extrême complexité de toute tentative de définition de la notion, ces deux éléments étant à l'origine d'autres flous puisque le mot est à l'origine de bon nombre de processus morphologiques de la langue, à commencer par le processus compositionnel. En revanche, Bauer note (p. 28) qu'il est indéniable que les mots composés se comportent comme des mots simples; le point faible de ce constat est que si l'on part de l'idée que le terme « mot » est difficile à définir et que le mot composé se comporte comme un mot, alors le mot composé sera également difficile à définir, ce qui amène Bauer à conclure que c'est certainement pour cela que les différentes études existantes peinent à se mettre d'accord sur une définition du mot composé.

3 Le deuxième point abordé par l'ouvrage Compounds and Compounding concerne la place de la syntaxe dans les constructions composées. Deux éléments clés dans la compréhension du fonctionnement intrinsèque des mots composés sont soulevés : la notion de noyau et l'idée de binarité dans la construction des mots composés. Premièrement, Bauer définit la notion de tête ou de noyau - head, en anglais. Bauer avance (p.40) que si la notion de noyau est régulièrement utilisée comme critère d'appartenance à la famille des mots composés en lien avec la notion d'hyponymie ( $a$ windmill is a mill, a drawbridge is a bridge), elle n'est peut-être pas d'une fiabilité à toute épreuve lorsque l'on travaille sur les mots composés, notamment parce que les manipulations mettant en avant le caractère hyponymique des constructions composées ont une efficacité limitée. Deuxièmement, l'idée selon laquelle un mot composé est une construction binaire est discutée. Le bilan que propose Bauer (p. 43) autour de cette question de la binarité est plutôt clair : il s'agit dans une très grande majorité des cas d'un critère qui fonctionne efficacement. Toutefois, il est à noter que Valerie Adams [2001], en raison du classement des mots composés coordonnés qu'elle propose, estime qu'il existe des composés non-binaires [2001:3]. Bauer soulève (p. 46) ensuite la question de la coexistence de deux notions dans les constructions composées : le processus compositionnel et la syntaxe sont vus par Bauer comme deux éléments qui cohabitent au sein des mots composés, que ce soit à gauche lorsque des syntagmes sont utilisés pour qualifier un objet, ou bien à droite comme éléments nonqualifiants.

4 Après avoir traité les questions de définition et de syntaxe, Bauer propose ensuite une analyse des valeurs sémantiques des mots composés, en soulignant les trois différentes manières qu'ont eues les linguistes de classer les mots composés à la lumière des relations sémantiques qu'ils contiennent: les études existantes se sont d'abord concentrées sur la grammaire sanskrite, puis ont travaillé sur le lien entre syntaxe et 
sémantique, et ont ensuite axé leur classification sur les différences entre composés endocentriques, exocentriques et coordinatifs. L'opposition entre composés endocentriques et composés exocentriques est, selon Bauer (p.64), l'une des classifications sémantiques des mots composés les plus majoritairement utilisées, notamment grâce à ses liens avec les notions de noyau et d'hyponymie, développées par Bauer dans la partie précédente de son ouvrage. Bauer estime (p. 106) que les valeurs sémantiques des mots composés sont très variables selon les types de composés utilisés (il passe en revue les valeurs sémantiques des noms composés, des adjectifs composés et des verbes composés), et selon les langues sur lesquelles on travaille (Bauer utilise des exemples de mots composés anglais, mais aussi allemands, danois, ou bien encore russes). Pour Bauer (p. 106), la difficulté à régir les relations sémantiques que l'on trouve au sein des mots composés participe à la difficulté avérée de décrire ces mots composés, notamment les noms, qui ont recours à un nombre très important et très différent de relations sémantiques.

5 Le quatrième chapitre de l'ouvrage est intitulé "The Classification of Compounds » (p. 107) : jusqu'à présent, Bauer a souligné à quel point les mots composés étaient difficiles à circonscrire, que ce soit d'un point de vue syntaxique ou sémantique. Dès lors, établir une typologie stable des mots composés semble délicat: Bauer annonce (p.107) d'emblée qu'il a remarqué, au cours de ses travaux sur les classifications existantes, un manque de cohésion évident entre les classifications qu'il a consultées. Bauer commente d'abord les classifications basées sur la grammaire sanskrite (p. 107), puis les travaux (p. 112) de Sergio Scalise et Antonietta Bisetto [2009]. Ces deux types de classification font appel aux valeurs sémantiques des mots composés, Bauer établit donc un lien entre cette partie de l'ouvrage et celle qui précède. Bauer clarifie la signification des termes issus de la grammaire sanskrite - tatpurusa, bahuvrihi, dvandva, avyayibhava - à la lumière de ceux utilisés par les linguistes anglophones - endocentric, exocentric, coordinative, subordinative. Bauer indique (p.110) que l'influence de la classification sanskrite n'est pas négligeable; cela étant dit, le point faible de la classification sanskrite est, toujours selon Bauer (p.110), qu'elle se fonde sur les comportements d'une langue de départ, qui sont ensuite appliqués à d'autres langues, ce qui pose des problèmes d'adaptation. Quant aux travaux de Scalise et Bisetto [2009], Bauer note (p.112) que l'axe principal utilisé dans leur classification est une frontière entre composés endocentriques et exocentriques. Bauer conclut son chapitre sur la classification des composés en argumentant (p.116) qu'une classification des mots composés étudiée seule ne prend véritablement de valeur que lorsqu'elle est comparée à d'autres classifications, qu'il s'agisse de classifications faisant usage d'autres critères d'organisation ou de classifications travaillant sur des langues différentes. Les analyses de Bauer sont suivies d'une tentative de classification des composés de l'anglais, fort utile pour quiconque s'intéresse au phénomène compositionnel: cette classification proposée par Bauer (p.120) est une application directe des théories proposées par Scalise et Bisetto [2009:50]. Cette classification utilise comme premier critère la distinction composé subordonné/composé coordonné puis comme deuxième critère la distinction composé exocentrique/composé endocentrique. Cette classification, qui prend en compte jusqu'à sept critères de distinction par classe de composés, est extrêmement riche et permet de lever de nombreuses ambiguïtés.

6 L'ultime chapitre de l'ouvrage est intitulé «Facets of English Compounding » (p. 126). Contrairement aux autres analyses mises en place par Bauer, ce chapitre s'intéresse uniquement aux composés en langue anglaise, et propose de dresser plusieurs bilans. 
Bauer propose par exemple de faire le point sur la question de l'accentuation des mots composés et sur la question des verbes à plusieurs éléments. À propos de l'accentuation, Bauer indique (p. 126) que même si elle est un outil qui permet de tracer des frontières entre les notions de «mot ", « séquence de mots » et «mot composé », il est délicat de considérer que l'accentuation puisse faire figure de facteur dans la définition du mot composé. Pour Bauer, cela est dû au fait que la place de l'accent dans un composé reste instable malgré l'existence de théories (Plag [2006], Giegerich [2009]) parvenant à prédire, en grande partie, la place de l'accent dans les composés. Ce chapitre aborde un autre point problématique: la question des verbes à plusieurs éléments, et la distinction entre ce que Bauer nomme des "phrasal verbs» (p.132) et des « compound verbs » (p. 136). Il s'agit d'une question épineuse qui met en évidence à la fois des problèmes de terminologie («phrasal verbs » ne fait pas l'unanimité) et des problèmes de morphologie (les "phrasal verbs" sont-ils plutôt des constructions syntaxiques ou des constructions morphologiques, s'interroge Bauer (p. 136)). Le bilan dressé par Bauer est contrasté, notamment lorsqu'il travaille sur des exemples issus de corpus (p. 140), et appelle à «further study» (p. 140). C'est d'ailleurs sur cette idée selon laquelle d'autres enquêtes devront être menées que ce chapitre se termine : bien que l'anglais soit une langue dont le fonctionnement a déjà été largement commenté et décrit, bien des pistes demeurent encore inexplorées. Bauer a travaillé sur la question de la composition en anglais pendant plusieurs décennies mais estime tout de même que « English compound still has much to teach us » (p. 167).

L'ouvrage Compounds and Compounding s'avère être un outil indispensable lorsque l'on travaille sur le processus compositionnel anglais, ou même plus globalement sur les questions de morphologie. Il permet de faire un point complet actualisé sur un sujet qui a pourtant déjà été traité à maintes reprises : c'est là toute la force de Bauer, qui, pour chaque point abordé, dresse un bilan des idées qui ont déjà été proposées pour mettre en lumière des idées nouvelles. On retiendra plus particulièrement le travail de Bauer à propos de la classification des mots composés, très souvent considéré comme un travail-clé dans la compréhension du processus compositionnel.

\section{BIBLIOGRAPHY}

ADAMS Valerie, 2001, Complex Words in English, Harlow: Pearson.

DI SCIULlo Anna Maria \& WILliams Edwin, 1987, On the Definition of Word, Cambridge: MIT Press.

GIEGERICH Heinz J., 2009, “The English compound stress myth” in Word Structure 2: 1-17.

PLAG Ingo, 2006, "The variability of compound stress in English: structural, semantic and analogical factors" in English Language and Linguistics 10: 143-172.

SCALISE Sergio \& BISETTO Antonietta, 2009, "The classification of compounds" in LIEBER Rochelle \& STEKAUER Pavol (Eds.), The Oxford Handbook of Compounding, Oxford: Oxford University Press: 34-53. 


\section{AUTHORS}

\section{JEAN MISSUD}

Jean Missud, Aix-Marseille Université, France.

Jean Missud est détenteur d'un Doctorat en Études Anglophones délivré par Aix-Marseille Université. Sa thèse de doctorat est intitulée « Analyse linguistique, stylistique et prosodique de l'adjectif composé anglais »; Jean Missud s'intéresse aux questions de morphologie, de syntaxe et de prosodie autour des mots composés (noms, adjectifs, verbes), ainsi qu'à leur place dans la littérature du monde anglophone. 